\title{
Short-Term Memory Demands of Reaction-Time Tasks That Differ in Complexity
}

\author{
Gordon D. Logan \\ Erindale College, University of Toronto, Ontario, Canada
}

\begin{abstract}
Four experiments were conducted to determine whether the increased reaction time produced by loading memory with a set of irrelevant items depended on the complexity of the stage structure or of the stimulus-response (S-R) mapping rules underlying the task. In each experiment, reaction-time tasks varying in complexity were performed alone and in the retention interval of a short-term memory task requiring ordered recall of eight digits. Experiment 1 compared simple, choice, and go/no-go tasks over 3 days of practice and found no interaction between memory load and task complexity. Experiment 2 replicated the first day of Experiment 1 with more power and again found no interaction. Since the different tasks required different numbers of stages, this ruled out stage structure as a factor loading memory. However, in Experiment 1 and 2, the same subjects performed all three tasks with the same set of letters and may have developed one comprehensive set of mapping rules for all three tasks, essentially eliminating differences in memory demands between tasks. Experiment 3 used different groups of subjects for a simple and a choice task with letters as stimuli to encourage the development of separate, differentially complex mapping rules for each task and found more interference in the choice task than in the simple task. Experiment 4 involved one group that performed the choice task with letters and the simple task with dots presented at different positions in the visual field, and another group that performed the simple task with letters and the choice task with dots. Comparisons within subjects between materials and between subjects within materials showed that with letters as stimuli, the choice task suffered more interference than the simple task. However, the choice task with dots showed no such pattern, perhaps because of high ideomotor compatibility. It is concluded that $\mathrm{S}-\mathrm{R}$ mapping rules are held in memory to enable performance on reaction-time tasks, and the findings are discussed in terms of strategies, preparation, and attentional control.
\end{abstract}

Reaction-time tasks that require no apparent storage of stimulus information suffer in terference when short-term memory is loaded with a set of irrelevant items. For example, the time to decide which of two lines is longer is increased substantially when the subject retains a set of eight consonants (Shulman \& Greenberg, 1971). This article reports an investigation of some aspects of reaction-time tasks that may be responsible for this interference.

The investigation began with the assumption that some aspects of reaction-time tasks demand access to the limited capacity of short-term memory and suffer interference when capacity is shared with a concurrent memory load. The more capacity required for the memory load, the less is available for the reaction-time task, so interference should increase with memory load. The available data support this assumption; for a variety of judgments, the impairment in reaction time is proportional to the load for loads of five items or more (Egeth, 1977; Logan, 1978; Shulman \& Greenberg, 1971; Shulman, Greenberg, \& Martin, 1971).

The same logic can be extended to 
Table 1

Reaction-Time Task Parameter That Interacted or Failed to Interact With Memory Load Organized According to Their Influence on Hypothetical Stages of Processing

\begin{tabular}{|c|c|c|}
\hline \multirow{2}{*}{$\begin{array}{l}\text { Stage of processing } \\
\text { affected }\end{array}$} & \multicolumn{2}{|c|}{ Effect of parameter } \\
\hline & Additivity & Interaction \\
\hline Encoding & $\begin{array}{l}\text { Brightness }^{\mathfrak{a}} \\
\text { Contrast }^{\mathrm{b}} \\
\text { Visual noise }\end{array}$ & Stimulus predictability ${ }^{\circ}$ \\
\hline Comparison & $\begin{array}{l}\text { Array size } \\
\text { Bar marker cues } \\
\text { Discriminability }\end{array}$ & $\begin{array}{l}\text { Stimulus predictability } \\
\text { Target set size } \\
\text { No. S-R alternatives }\end{array}$ \\
\hline Decision & Decision type (yes-no) & \\
\hline Response selection & $\begin{array}{l}\text { Response type (vocal vs. manual) } \\
\text { 2-choice verbal S-R compatibility } \\
\text { 2-choice spatial S-R compatibility }\end{array}$ & $\begin{array}{l}\text { Stimulus predictability } \\
\text { No. } S-R \text { alternatives } \\
10 \text {-choice spatial } S-R \text { compatibility }\end{array}$ \\
\hline
\end{tabular}

Note. The results are from Logan (1978) unless indicated otherwise. S-R = stimulus-response. a Egeth (1977). b Logan (Note 1). ${ }^{\circ}$ Keele and Boies (1973). ${ }^{d}$ Sternberg (1969) and Logan (1978). ${ }^{\circ}$ Logan (1979). f Crowder (1967).

develop a procedure for identifying the demanding aspects: For a given memory load, versions of a reaction-time task that demand more memory capacity should suffer more interference than versions that demand less memory capacity. This suggests a two-factor experiment (at least) in which the presence or absence of a substantial memory load is crossed with some parameter affecting the reaction-time task. If the parameter varies the demand that the task places on short-term memory, more interference should be observed at some parameter values than at others; that is, the parameter should interact with

This research was supported by Grant A0682 from the Natural Sciences and Engineering Research Council of Canada. A shorter report was presented at the annual meeting of the Canadian Psychological Association, Vancouver, British Columbia, June 1977.

I am grateful to Rick Kantor, Paul Manchester, and Lee Smith for running the subjects. I would like to thank Ron Fisher, Morris Moscovitch, and Jane Zbrodoff for valuable discussion and criticism, and two anonymous reviewers for helpful comments on the manuscript.

Requests for reprints should be sent to Gordon D. Logan, Department of Psychology, Erindale College, University of Toronto, Mississauga, Ontario, L5L 1C6, Canada. memory load. Alternatively, if the parameter is unrelated to the memory demands of the task, the same amount of interference should be observed for all parameter values; that is, the parameter should not interact with memory load. Thus, in a complex experiment involving memory load, the pattern of interaction and additivity should reveal which aspects of the reaction-time task demand short-term memory (also see Egeth, 1977 ; Logan, 1978, 1979).

Previous investigations using this logic found that many of the parameters typically varied in reaction-time tasks do not interact with memory load (e.g., Egeth, 1977 ; Logan, 1978; Logan, Note 1). Of 14 parameters investigated, only 4 produced interactions. All 14 appear in Table 1, organized according to their influence on the four stages of processing believed to underly performance in choice-reactiontime tasks (e.g., Smith, 1968; Sternberg, 1969). This organization makes two points clear: First, the widespread additivity indicates that there are other limitations on performance besides short-term memory capacity; short-term memory is not responsibile for all the processing that underlies performance. Second, finding evidence for interaction and additivity at each stage 
of processing indicates that short-term memory is not another stage of processing like encoding, comparison, and so on, through which all information flows. Its role is more subtle than that.

The present investigation explored the possibility that short-term memory holds together the components of reaction-time tasks to make performance possible. Most reaction-time tasks studied in the laboratory require a novel combination of abilities that have been practiced extensively in separate contexts in the natural environment (e.g., pressing buttons upon seeing letters). Short-term memory may be important in maintaining the required organization until a response can be emitted (Logan, 1978, 1979; also see Doll, 1969; Klapp, 1976). I consider two specific interpretations of this view, both of which account for the findings summarized in Table 1.

First, in terms of traditional stage analysis, the abilities may be viewed as processing stages, so that short-term memory retains the configuration of stages required for the task. Since different tasks may involve different stages or different numbers of stages, this function is logically necessary. The parameters that interact with memory load may represent "connections" between stages, specifying how the output from one stage is to be used as input by another. (Perhaps this is why two of the parameters that produced interactions each influenced more than one stage.) The parameters whose effects were additive with respect to memory load represent complexities within stages that may not affect how stages are combined.

This interpretation leads directly to an experimental test: If short-term memory is used to hold stages together, tasks with more stages should suffer more interference from a concurrent memory load than tasks with fewer stages; memory load should interact with task complexity. Alternatively, if stages are not held in memory, tasks with different numbers of stages should suffer the same amount of interference; memory load and task complexity should have additive effects. These predictions can be tested readily by compar- ing the interference produced by a standard (eight-digit) memory load in three reactiontime tasks : simple tasks, which require one response to all stimuli, go/no-go tasks, which require one response to half of the stimuli and no response to the other half, and choice tasks, which require one response to half of the stimuli and another response to the other half. Traditional stage theory (e.g., Donders, 1868/1969; Woodworth, 1938 ) holds that simple tasks involve two stages (perception and response), that go/no-go tasks involve three stages (perception, discrimination, and response), and that choice tasks require four stages (perception, discrimination, response selection, and response). Thus, if stages are held together in short-term memory, simple tasks should suffer less interference than go/no-go tasks, and go/no-go tasks should suffer less interference than choice tasks. Moreover, this prediction should hold in all situations in which different tasks involve different numbers of stages. Note, however, that this prediction addresses only the possibility that the stages defined by traditional stage theory are held in memory; failure to find interactions does not rule out other interpretations of stage theory, either as processing structures or as control structures held in short-term memory. Further, failure to find interactions does not rule out the traditional stages as possible processing structures; performance could be based on stages but controlled by retaining something other than stages in short-term memory.

The second interpretation also predicts an interaction between memory load and task complexity but does so without the assumptions of traditional stage theory so that interactions may appear in some situations but not others. It rests on the assumption that subjects remember the relevant stimulus-response (S-R) mapping (assignment of stimuli to responses) rather than stages, and that this by itself may be sufficient to recruit and organize the required abilities. Since S-R mapping changes from situation to situation, this function is logically necessary (if stage theory is not assumed). The parameters in 
Table 2

Production Systems Representing Mapping Rules for Simple, Go/No-Go, and Choice Reaction-Time Tasks

\begin{tabular}{|c|c|c|c|}
\hline Task & Production & Condition $\rightarrow A c$ & tion \\
\hline Simple (A) & A1 & (TASK $=\mathrm{A} \& \mathrm{EYE}=$ something & $\rightarrow$ HAND $=$ RIGHT $)$ \\
\hline Choice (B) & $\begin{array}{l}\text { B1 } \\
\text { B2 } \\
\text { B3 } \\
\text { B4 }\end{array}$ & $\begin{array}{l}(\mathrm{TASK}=\mathrm{B} \& \mathrm{EYE}=\mathrm{E} \\
(\mathrm{TASK}=\mathrm{B} \& \mathrm{EYE}=\mathrm{F} \\
(\mathrm{TASK}=\mathrm{B} \& \mathrm{EYE}=\mathrm{H} \\
(\mathrm{TASK}=\mathrm{B} \& \mathrm{EYE}=\mathrm{L}\end{array}$ & $\begin{array}{l}\rightarrow \text { HAND }=\text { RIGHT }) \\
\rightarrow \text { HAND }=\text { RIGHT }) \\
\rightarrow \text { HAND }=\text { LEFT }) \\
\rightarrow \text { HAND }=\text { LEFT })\end{array}$ \\
\hline Go/no-go (C) & $\begin{array}{l}\mathrm{C} 1 \\
\mathrm{C} 2\end{array}$ & $\begin{array}{l}(\mathrm{TASK}=\mathrm{C} \& \mathrm{EYE}=\mathrm{E} \\
(\mathrm{TASK}=\mathrm{C} \& \mathrm{EYE}=\mathrm{F}\end{array}$ & $\begin{array}{l}\rightarrow \text { HAND }=\text { RIGHT) } \\
\rightarrow \text { HAND }=\text { RIGHT) }\end{array}$ \\
\hline $\begin{array}{l}\text { Simple, choice, } \\
\text { and go/no-go }\end{array}$ & $\begin{array}{l}\text { D1 } \\
\text { D2 } \\
\text { D3 } \\
\text { D4 } \\
\text { D5 } \\
\text { D6 }\end{array}$ & $\begin{array}{l}\text { (TASK }=\text { anything } \& \mathrm{EYE}=\mathrm{E} \\
\text { (TASK }=\text { anything \& } \mathrm{EYE}=\mathrm{F} \\
\text { (TASK }=\mathrm{A} \& \mathrm{EYE}=\mathrm{H} \\
\text { (TASK }=\mathrm{A} \& \mathrm{EYE}=\mathrm{L} \\
\text { TASK }=\mathrm{B} \& \mathrm{EYE}=\mathrm{H} \\
(\mathrm{TASK}=\mathrm{B} \& \mathrm{EYE}=\mathrm{L}\end{array}$ & $\begin{array}{l}\rightarrow \text { HAND }=\text { RIGHT) } \\
\rightarrow \text { HAND }=\text { RIGHT) } \\
\rightarrow \text { HAND }=\text { RIGHT }) \\
\rightarrow \text { HAND }=\text { RIGHT }) \\
\rightarrow \text { HAND }=\text { LEFT }) \\
\rightarrow \text { HAND }=\text { LEFT })\end{array}$ \\
\hline
\end{tabular}

Note. The systems assume a visual processing system, EYE, that registers symbolic events like letters or the presence of a stimulus; a response system, HAND, that accepts and executes symbolic commands which result in overt responses; and a control variable, TASK, to distinguish experimental conditions. $\mathrm{A}=$ simple $\mathrm{RT}, \mathrm{B}=$ choice $\mathrm{RT}, \mathrm{C}=$ go/no-go $\mathrm{RT}$, and $\&=$ logical conjunction.

Table 1 that interacted with memory load seem to reflect the number, complexity, or priority of different $\mathrm{S}-\mathrm{R}$ mappings, whereas the parameters whose effects were additive seem to reflect stimulus properties like signal strength. (The two-choice S-R compatibility effects are an exception, but may not replicate with greater degrees of choice; e.g., Crowder, 1967.)

This interpretation also predicts an interaction between memory load and task complexity, since simple tasks can involve fewer mapping rules than go/no-go tasks, and go/no-go tasks can involve fewer mapping rules than choice tasks. But the interaction need not be predicted in all situations, since in principle simple tasks can involve as many mapping rules as choice tasks or go/no-go tasks. To illustrate those possibilities, Table 2 contains S-R mapping rules for the three tasks represented as production systems. ${ }^{1}$ Production Systems A, B, and C represent the former situation in which a minimal set of mapping rules is used for each task; Production System D represents the latter situation in which one larger set of mapping rules is used for all three tasks. Production Systems $A, B$, and $C$ would produce interactions between memory load and task complexity; Production System D would not. ${ }^{2}$ Thus,

${ }^{1}$ A production system is a set of condition $\rightarrow$ action rules in which an action is taken when the corresponding condition is satisfied. Usually, productions are assumed to be stored in long-term memory (e.g., Newell, 1973). I assume that productions are held in short-term memory when they are first acquired and are transferred to long-term memory after they have been debugged and practiced.

${ }^{2}$ In Production System D, the same mapping rules could produce different reaction times in the different tasks because confusions among stimuli have different consequences in different tasks. In choice tasks, confusions lead to errors; in simple tasks, they lead to correct responses. Assume that evidence for each condition accumulates over time and that a condition is satisfied when the evidence for it exceeds a threshold. Assume that the thresholds are lowered when the response is judged to be correct and raised when the response is judged to be in error (i.e., P1: (FEEDBACK $=$ CORRECT $\rightarrow$ LOWER THRESHOLD); P2: (FEEDBACK $=$ ERROR $\rightarrow$ RAISE THRESHOLD)). In choice tasks, repeated application of this scheme would lower the thresholds for each letter to a point just above the noise level produced by the presentation of other letters. In simple tasks, repeated application of the same scheme would lower the thresholds for each letter to a point just above the noise level produced by no stimulation. Since the noise from presentation of other letters should be greater than the noise from presentation of no letters, choice tasks should require more 
the interpretation based on traditional stage theory can be distinguished from this one, based on S-R mapping, by finding conditions under which subjects will adopt a production system like $D$ rather than ones like $\mathrm{A}, \mathrm{B}$, and $\mathrm{C}$.

Previous research with concurrent memory loads suggests that subjects develop minimal sets of mapping rules under conditions in which stimuli are mapped consistently onto the same responses, and that subjects maintain complex sets of mapping rules (i.e., one rule is maintained in memory for each target item or S-R alternative) under conditions in which stimuli are mapped onto different responses from the same set from trial to trial or day to day (i.e., varied mapping; see Logan, 1978, 1979). After practice with consistent mapping, the greater interference found initially with larger target sets or larger sets of S-R alternatives disappeared; the sets became chunked or automatized, and the larger ones suffered no more interference than the smaller ones. After practice with varied mapping, however, the greater interference with larger sets remained; no chunking or automatization was apparent.

Thus, in the present context with tasks that vary in complexity, we might expect subjects to develop minimal mapping rules (i.e., Production Systems A, B, and C) when different tasks use different stimuli and responses and maintain the mapping consistently throughout practice, or when the different tasks are performed by different subjects. Under these conditions, interactions between memory load and task complexity should appear. Furthermore, we might expect subjects to develop complex mapping rules (i.e., Production System D) when tasks of different complexity use the same stimuli and responses but vary the mapping, and when the different tasks are performed by the same subject. Under these conditions, the effects of memory load and task complexity should be additive.

evidence than simple tasks and so should produce longer reaction times, even with the same mapping rules.
Four experiments were conducted to explore these possibilities. The first two were designed with only stage theory in mind, and varied task complexity within subjects by varying the mapping between one set of stimuli and one set of responses. The in terpretation in terms of S-R mapping rules was developed when the first two experiments failed to produce interactions, and led to the third and fourth experiments in which task complexity was varied between subjects and between stimulus sets within subjects.

\section{Experiment 1}

This experiment examined performance on simple, go/no-go, and choice reaction time tasks with and without an eight-digit memory load over a period of 3 days. Previous research had suggested that much of the reduction in memory-load interactions is completed in 3 days of practice (Logan, 1978, 1979). In this experiment, the same stimuli (the letters $E, F, H$, and L) were used for all three tasks, and each subject performed every task.

\section{Method}

Subjects. Twelve undergraduate students from Erindale College were paid for participating in three 1-hr sessions.

Apparatus and stimuli. The stimuli for the reaction-time task were presented visually using a Carousel slide projector (Kodak Model 650H) equipped with a tachistoscopic shutter (Uniblitz Model 26) under the control of Grason-Stadler logic and timing devices. A second projector equipped with a similar shutter exposed a fixation point whenever the stimuli were not exposed. The stimuli were the letters E, F, H, and L. Each one was mounted centrally on a glass slide using black uppercase Letraset (1570) and rear projected on a translucent screen. Viewed at a distance of $75 \mathrm{~cm}$ (using a headrest), a letter subtended about $.53^{\circ}$ of visual angle vertically and about $.45^{\circ}$ horizontally. The letter appeared centered on a white field subtending $6.49^{\circ}$ of visual angle vertically and $9.93^{\circ}$ horizontally. The luminance of stimulus and fixation fields was approximately $48 \mathrm{~cd} / \mathrm{m}^{2}$. The letters were exposed for $500 \mathrm{msec}$.

The stimuli for the memory task were random strings of eight unique digits presented at a rate of $.75 \mathrm{sec}$ per digit. Each string was preceded by a spoken ready signal ("ready") that occurred 1.5 sec before the first digit. The strings were recorded 


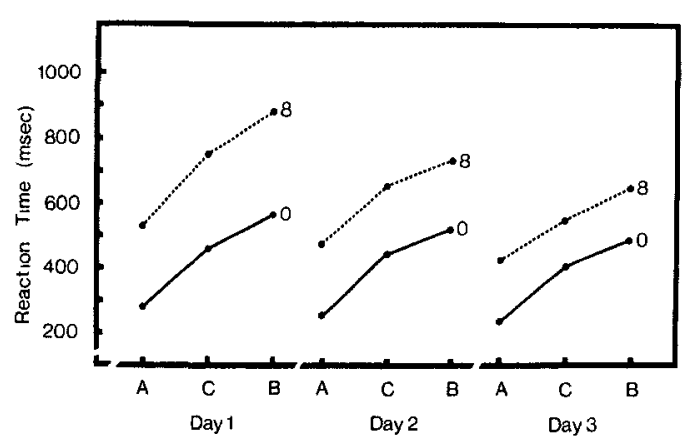

Figure 1. Mean reaction times in simple (A), choice (B), and go/no-go (C) tasks each day in Experiment 1. (Memory load is the parameter.)

in a male voice and were played back through a speaker at a comfortable listening level. A different list was prepared for each trial.

Recall was signaled by a $1000-\mathrm{Hz}, 500$-msec tone presented $5 \mathrm{sec}$ after the stimulus turned off.

Procedure. Dual-task trials consisted of the following series of events: (a) the presentation of a digit string, (b) the presentation of a letter, (c) the response to the letter, (d) the presentation of a tone signaling recall, and (e) the attempt to recall the string in the order in which it was presented. Single-task reaction-time trials omitted Events a, $d$, and $\mathrm{e}$; single-task memory trials omitted Events $b$ and $c$ but kept the same retention interval $(6.5 \mathrm{sec})$.

Each session began with 12 single-task memory trials, followed by 144 single- and dual-task reactiontime trials and ended with 12 single-task memory trials. In the reaction-time trials, each subject completed two blocks of 24 trials with each reactiontime task (one in single- and one in dual-task conditions) before moving on to the next. Half of the subjects had dual-task conditions in the first block with each task and single-task conditions in the second for Days 1 and 3 , and the reverse on Day 2. The other half had single-task conditions in the first block and dual-task conditions in the second for Days 1 and 3, and the reverse on Day 2 . The order of reaction-time tasks varied within subjects over sessions according to a Latin square. Each day, each of the six possible orders of tasks was assigned to two subjects.

Thirty-six copies of each of the four slides $(\mathrm{E}, \mathrm{F}$, $H$, and $L$ ) were made to construct six trays of 24 trials. These trays were presented in the same order each day, but because of the balancing of orders of tasks, each tray was used equally often with each task in single- and dual-task conditions.

In the choice task, half of the subjects pressed the right button if $\mathrm{E}$ or $\mathrm{L}$ appeared and the left button if $\mathrm{F}$ or $\mathrm{H}$ appeard. The other half pressed right for $E$ or $F$ and left for $H$ or L. In the go/no-task, each subject pressed the right button to the two letters assigned to it in the choice task and made no response to the other two letters. In the simple task, each subject pressed the right button for all four letters. The assignment of letters to buttons in the choice task was orthogonal to the assignment to orders of performing the tasks; one subject with each letter-button assignment received one of the six possible orders of tasks each session.

The instructions, given in detail in the first session and reviewed in the second and third, stressed speed and accuracy in the reaction-time tasks and accuracy in the memory task. Subjects were asked to recall the memory digits in the order in which they were presented. The memory task was defined as primary and the reaction-time tasks as secondary in two ways: First, subjects were given feedback about their performance on the memory task; they were told the number and nature of their errors after each trial. They were given no feedback about speed or accuracy in the reaction-time task. Second, subjects were told to consider the memory task the more important of the two and to maximize their performance on it in all conditions (cf. Posner \& Boies, 1971).

During testing, the room was dimly lit by a reading lamp and the ambient light from the projectors. Each day the time taken for instructions served as a dark adaptation period.

\section{Results}

Reaction-time tasks. Each day, each subject completed 24 trials in each combination of reaction-time task and memory load conditions. Mean reaction times were computed for correct responses in each of these conditions for each subject, and these scores were submitted to an analysis of variance. The means across subjects appear in Figure 1.

Reaction time increased with task complexity, $F(2,22)=82.27, p<.01$; performance with the simple task was faster than with go/no-go, and go/no-go was faster than choice. Retaining eight digits also increased reaction time, $F(1,11)$ $=53.33, p<.01$, but overall the increase was the same for each task; the crucial interaction between load and tasks was not significant, $F(2,22)<1$. Inspection of Figure 1 might suggest that the memory load effect increased with task complexity on Day 1 but not on subsequent days after performance had automatized somewhat. Indeed, both reaction time and the memory-load effect diminished with practice, $F_{\mathrm{s}}(2,22)=15.57$ and 5.30 , respectively, $p<.05$, suggesting automatization. However, there was no evidence of a differential effect between tasks. Neither the interac- 
Table 3

Proportion of Errors in Each Reaction-Time Task for Each Day of Experiment 1 and for the One Day of Experiment 2

\begin{tabular}{|c|c|c|c|c|c|c|}
\hline \multirow[b]{2}{*}{ Experiment } & \multirow[b]{2}{*}{ Day } & \multirow{2}{*}{$\begin{array}{c}\text { Memory } \\
\text { load }\end{array}$} & \multicolumn{4}{|c|}{ Reaction-time task } \\
\hline & & & None & A & $\mathrm{C}$ & B \\
\hline \multirow[t]{3}{*}{1} & 1 & $\begin{array}{l}0 \\
8\end{array}$ & $-\overline{(5.86)}$ & $\begin{array}{r}.004 \\
.008 \\
(5.96)\end{array}$ & $\begin{array}{r}.017 \\
.017 \\
(5.85)\end{array}$ & $\begin{array}{r}.033 \\
.029 \\
(6.02)\end{array}$ \\
\hline & 2 & $\begin{array}{l}0 \\
8\end{array}$ & $-\overline{(6.53)}$ & $\begin{array}{r}.017 \\
.004 \\
(6.37)\end{array}$ & $\begin{array}{r}.000 \\
.000 \\
(6.31)\end{array}$ & $\begin{array}{r}.021 \\
.021 \\
(6.38)\end{array}$ \\
\hline & 3 & $\begin{array}{l}0 \\
8\end{array}$ & $-\overline{(6.96)}$ & $\begin{array}{r}.003 \\
.000 \\
(6.77)\end{array}$ & $\begin{array}{r}.000 \\
.000 \\
(6.81)\end{array}$ & $\begin{array}{r}.013 \\
.008 \\
(6.66)\end{array}$ \\
\hline 2 & 1 & $\begin{array}{l}0 \\
8\end{array}$ & $-\overline{(6,14)}$ & $\begin{array}{r}.016 \\
.017 \\
(5.75)\end{array}$ & $\begin{array}{r}.031 \\
.097 \\
(5.88)\end{array}$ & $\begin{array}{r}.057 \\
.068 \\
(5.88)\end{array}$ \\
\hline
\end{tabular}

Note. Simple $=A$, choice $=$ B, go $/$ no-go $=$ C. Recall accuracy is in parentheses.

tion between memory load, tasks, and days, $F(4,44)<1$, nor a contrast comparing the memory load effect from the choice task with the effect from the simple task on Day $1, F(1,22)=2.82, p>.10$, were significant.

The proportion of errors in each condition each day appears in Table 3 . The pattern of these data reflects the trends in the reaction-time data.

Memory task. Each day each subject recalled 24 lists of eight digits in each of three dual-task conditions (i.e., with the simple, go/no-go, and choice task) and in two 12-trial single-task blocks. Single-task blocks were collapsed, and the mean number of digits recalled in each condition (one single task and three dual task) was computed for each subject. These data were submitted to an analysis of variance. Means across subjects appear in Table 3.

Recall accuracy increased over days, $F(2,22)=22.86, p<.01$, but was the same for each task, $F(3,33)<1$. Moreover, the interaction between tasks and days was not significant, $F(6,66)<1$.

\section{Discussion}

This experiment provided no evidence that reaction-time tasks that differ in complexity demand different amounts of memory capacity. Before accepting the null hypothesis of no interaction, however, I thought it was important to examine more data, particularly in view of the suggestion of an interaction between memory load and task complexity on Day 1 when the tasks were first introduced. For this reason, the second experiment was conducted.

\section{Experiment 2}

This experiment was an exact replication of Experiment 1 except for the following changes: (a) Only Day 1 was replicated, since the crucial effects in Experiment 1 seemed to appear only on the first day; (b) four "catch" trials involving blank slides were inserted in each block of 24 trials to prevent anticipatory responding; and (c) the number of subjects employed was doubled.

\section{Method}

Subjects. Twenty-four undergraduate students and laboratory staff from Erindale College volunteered their services without pay for one 1-hr session.

Apparatus and stimuli. The visual stimuli were the same as those used in Experiment 1 except that four catch trials were added to each block of 24 reaction-time trials, increasing the number of trials 


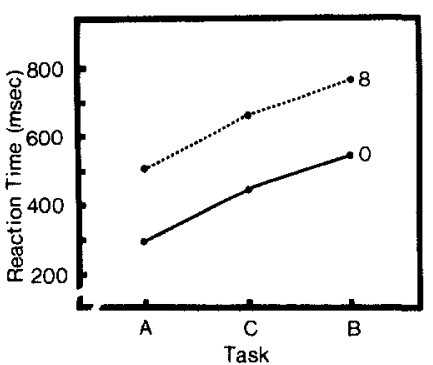

Figure 2. Mean reaction times in simple (A), choice (B), and go/no-go (C) tasks in Experiment 2. (Memory load is the parameter.)

per block to 28. Blank slides were used as stimuli on catch trials to eliminate differences in the sounds emanating from the projector. The memory stimuli were the same except that four additional lists were used in each dual-task block.

Procedure. The procedure was the same as in Experiment 1. In addition, subjects were told about the catch trials and were told not to respond to them. On dual-task trials, however, they were instructed to recall the memory load as usual.

As in Experiment 1, all three reaction-time tasks used the same stimuli ( $E, F, H$, and $L$ ), and each subject performed every task.

\section{Results}

Reaction-time tasks. Each subject completed 24 trials in each combination of tasks and memory-load conditions. Mean correct reaction times were computed and analyzed as before and appear in Figure 2.

As in Experiment 1, reaction time increased with task complexity, $F(2,46)$ $=101.18, p<.01$, and with memory load, $F(1,23)=75.28, p<.01$, but again, the interaction between them was not significant, $F(2,46)<1$. Neither was a contrast comparing the memory-load effect in the choice task with the one in the simple task, $F(1,46)<1$.

The proportion of errors in each condition appears in Table 3. Again, the trends reflect the pattern of the reaction-time data.

Memory task. Each subject recalled 24 lists of eight digits in single-task conditions and with each reaction-time task in dualtask conditions. Means in each combination of conditions were computed for each subject, and the means across subjects are displayed in Table 3.

In an analysis of variance performed on these data, conditions were found to differ significantly, $F(3,69)=5.06, \quad p<.01$. Orthogonal contrasts showed that recall was better in single-task conditions that in dual-task conditions, $F(1,69)=13.59$, $p<.01$, but there were no significant differences between dual-task conditions.

\section{Discussion}

Neither experiment showed evidence that more complex tasks suffer more interference from a concurrent memory load. In both experiments, the main effects of memory load and task complexity were highly significant, yet the $F$ ratios for their interactions were less than unity. The null hypothesis of no interaction seems the best description of the data.

These findings are inconsisent with the view that to enable performance on a task, subjects hold in memory the stages defined by traditional stage theory. That view predicts an interaction between memory load and task complexity under all conditions, since simple tasks always require fewer stages than go/no-go tasks, and go/no-go tasks always require fewer stages than choice tasks. However, the findings are consistent with the alternate view that $\mathrm{S}-\mathrm{R}$ mapping rules are held in memory to enable performance (or with other interpretations of stage theory). The mapping of letters onto response buttons varied between tasks within subjects, and this may have led subjects to adopt an all-encompassing set of rules, like Production System D, which could handle all three tasks without changing. Alternatively, they may have remembered the mapping of each letter onto each response in each task (i.e., using Production System B, and changing the values of the response variables for each task), holding constant the complexity of mapping rules.

There are two difficulties with this interpretation. First, the findings confirm only half of the prediction, namely, additive effects with varied mapping. The interactions predicted with consistent mapping remain to be demonstrated. They are particularly important because the ob- 
served additivity is also consistent with the view that interactions will never be obtained (cf. Egeth, 1977). Second, the interpretation in terms of $S-R$ mapping rules was developed after the results were obtained, so it is not surprising that the interpretation accounts for them. To be acceptable, it must account for effects not yet observed, namely, the interactions under consistent mapping conditions. For these reasons, the remaining two experiments were conducted.

\section{Experiment 3}

In this experiment, consistent mapping was ensured by having one group of subjects perform a simple task and another group perform a choice task. Both groups used the same stimuli (letters). To determine whether subjects in the two groups did develop different mapping rules (i.e., Production Systems A and B), the number of letters relevant on a trial (two or four) was varied within subjects in both tasks. In simple task, all letters in the two sets mapped consistently onto the same response; in the choice task, half of the letters in each set were mapped consistently onto one response, and the other half were mapped consistently onto the other. Number of letters was not expected to influence performance in the simple task because subjects were not expected to remember a separate mapping rule for each letter (i.e., Production System A would suffice for both sets of letters). By contrast, number of letters was expected to affect performance in the choice task by increasing the level of discrimination required and by increasing the number of $S-R$ mapping rules to be remembered. From previous findings, the former was expected to produce larger reaction times with four letters than with two (Sternberg, 1969), and the latter was expected to produce greater interference from a memory load with four letters than with two (Logan, 1978). Thus, the important interactions are between number of letters and tasks to demonstrate the effects of different mapping rules on performance and between

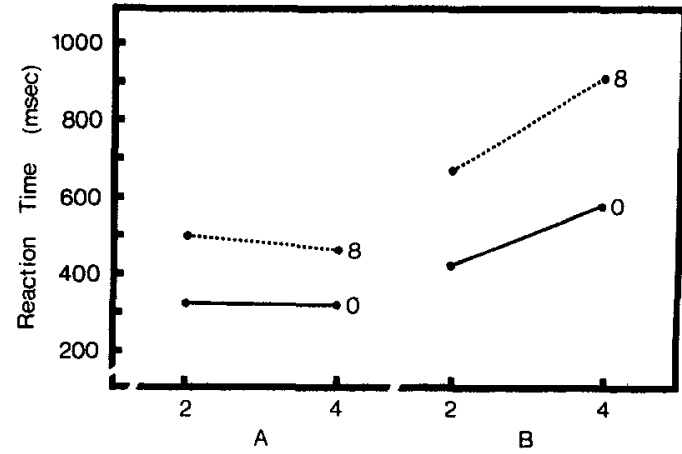

Figure 3, Mean reaction times in simple (A) and choice (B) tasks as a function of the number of letters relevant on a trial. (Memory load is the parameter.)

tasks and memory load and between number of letters, tasks, and memory load to demonstrate the effects of different mapping rules on memory.

\section{Method}

Subjects. Thirty-two undergraduate students and laboratory staff from Erindale College volunteered their services for one 1-hr session without pay.

Apparatus and stimuli. The digit strings for the memory task were the ones used in the previous experiments. Two sets of letters were used in the reaction-time tasks: The four-letter set was the one used in the previous experiments $(\mathrm{E}, \mathrm{F}, \mathrm{H}$, and $\mathrm{L})$. The two-letter set contained the letters $E$ and $H$, made from the same Letraset in the same manner as the four-letter set.

Procedure. The experiment consisted of 12 single-task memory trials, 112 single- and dual-task reaction-time trials (divided into four blocks of 28 trials), and 12 single-task memory trials. The 28 trials in each reaction-time block involved 24 letter trials and 4 catch trials. Each subject began with one letter set (half began with four and half with two) and completed two blocks with it (one with memory load, one without) before moving on to the next. Half of the subjects (16) performed the simple task only, and half performed the choice task only.

In the simple task all letters from the two sets were mapped onto the right button. In the choice task, $E$ and $F$ were always mapped onto the right button, and $H$ and $L$ were always mapped on to the left.

\section{Results}

Reaction-time tasks. Each subject in each group completed 24 trials with each letter set in single- and dual-task conditions. 
Table 4

Proportion of Errors in Each Reaction-Time Task as a Function of Number of Letters Relevant on a Trial and Recall Accuracy in Each

Condition of the Memory Task in Experiment 3

\begin{tabular}{|c|c|c|c|c|}
\hline \multirow{2}{*}{$\begin{array}{l}\text { Reaction- } \\
\text { time task }\end{array}$} & \multirow{2}{*}{$\begin{array}{l}\text { Memory } \\
\text { load }\end{array}$} & \multicolumn{3}{|c|}{ No. letters relevant } \\
\hline & & None & 2 & 4 \\
\hline A & $\begin{array}{l}0 \\
8\end{array}$ & $-\overline{(5.71)}$ & $\begin{array}{r}.060 \\
.036 \\
(5.16)\end{array}$ & $\begin{array}{r}.049 \\
.026 \\
(5.19)\end{array}$ \\
\hline B & $\begin{array}{l}0 \\
8\end{array}$ & $-\overline{(5.64)}$ & $\begin{array}{r}.057 \\
.034 \\
(5.50)\end{array}$ & $\begin{array}{r}.070 \\
.125 \\
(5.30)\end{array}$ \\
\hline
\end{tabular}

Note. Simple $=$ A, choice $=$ B. Recall accuracy is in parentheses.

Mean reaction time for correct responses was computed for each subject and submitted to an analysis of variance. Means across subjects are presented in Figure 3.

As the figure shows, subjects performing the simple task were considerably faster than subjects performing the choice task, $F(1,30)=45.13, p<.01$, and overall, reaction time was longer with a memory load than without, $F(1,30)=66.26$, $p<.01$. As expected, subjects performing the choice task suffered more from loading than subjects performing the simple task; the interaction between memory load and tasks was significant, $F(1,30)=5.54$, $p<.05$.

Overall, reaction time was longer with the four-letter set than with the two-letter set, $F(1,30)=50.26, p<.01$, and the effect was considerably stronger for the choice group than for the simple group, $F(1,30)=76.29, p<.01$. The interaction between number of letters, tasks, and memory load was significant, $F(1,30)=$ $4.55, p<.05$, in a way that suggested that the memory-load effect increased with number of letters in the choice group but not in the simple group (see Figure 3 ). However, analyses within each task suggested that the three-way interaction reflected two opposing two-way interactions: In the simple task, the memory-load effect tended to be smaller with four letters than with two, $F(1,15)=2.68, p<.15$, whereas in the choice task, it tended to be larger with four letters than with two, $F(1,15)=2.49, p<.15$. The error data are presented in Table 4.

Memory task. Each subject in each group recalled 24 eight-digit lists in singletask conditions and with two and four letters relevant in dual-task conditions. Means were computed in each of these conditions for each subject, and the means across subjects appear in Table 4 .

Analysis of variance revealed no significant differences between groups, $F(1,30)$ $<1$, but there were significant differences between conditions, $F(2,60)=6.24, p<$ .01 , such that recall was better in single-task conditions than in dual-task conditions, $F(1,60)=12.03, p<.01$, but did not differ between dual-task conditions.

\section{Discussion}

This experiment found a larger effect of memory load in a choice task than in a simple task, contrary to the findings of Experiments 1 and 2. In those experiments, simple and choice tasks were performed by the same subjects, presumably using the same complex set of mapping rules (i.e., Production System D). In Experiment 3, however, simple and choice tasks were performed by different subjects, presumably using different mapping rules (i.e., Production Systems A and B). The interaction suggests that the rules used for the simple task were less complex than those used for the choice task.

Further evidence that subjects in the different groups remembered different sets of mapping rules is available in the effects of the number of letters relevant on a trial. Number of letters had a large effect in the choice group but not in the simple group, suggesting that the choice group used more complex mapping rules. The tendency for memory load to have a stronger effect with more letters in the choice group but not in the simple group suggests that the choice group remembered more complex mapping rules as well. This conclusion is weakened somewhat by the nonsignificance of the within-subject (within-task) interactions 
between number of letters and memory load.

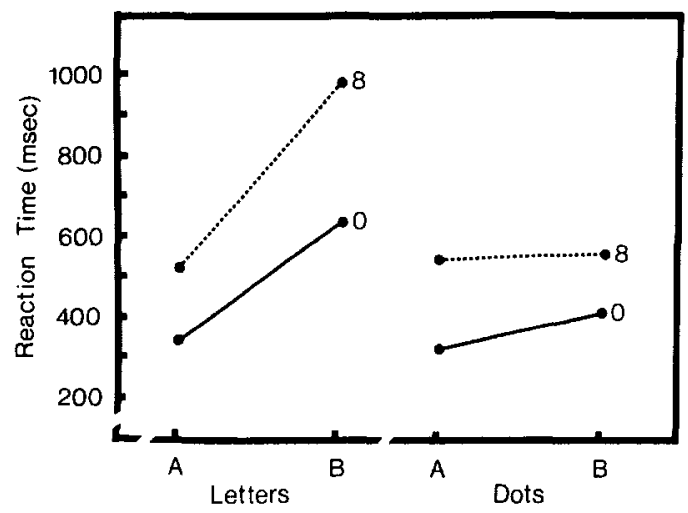

The previous experiment ensured consistent mapping by having different subjects perform the different tasks. Experiment 4 ensured consistent mapping by having the same subjects perform simple and choice tasks using a different set of stimuli for each task. Each subject performed the simple task with one kind of stimulus (e.g., dots) and the choice task with the other (e.g., letters). In both tasks, stimuli were mapped consistently onto responses. Moreover, instructions for the two tasks were given separately; instructions for the second task were not given until the first task was completed. Under these conditions, subjects were expected to adopt a separate set of mapping rules for each task, and the sets were expected to differ in complexity and memory demands, so that memory-load effects would be larger in the choice task than in the simple task. The design allowed this prediction to be evaluated within subjects, between materials and between subjects, within materials.

\section{Method}

Subjects. Thirty-two undergraduate students and laboratory staff from Erindale College volunteered their services without pay for one 1-hr session.

Apparalus and stimuli. The digit lists for the memory task and the letter stimuli for the reactiontime tasks were the same as those used in Experiments 1 and 2 . The dot stimuli for the reaction-time tasks were made by mounting black dots (Letraset 553) on glass slides. One dot appeared on each slide in one of the four corners of an imaginary rectangle centered on the fixation point. Each dot subtended about $.61^{\circ}$ of visual angle in diameter, and the imaginary rectangle subtended about $3.21^{\circ}$ vertically and $5.19^{\circ}$ horizontally.

Procedure. The procedure was the same as in the previous experiments with three exceptions: First, in the choice task with dots, subjects were told to press the left button to the top-left and bottom-left dot and the right button to the top-right dot and bottom-right dot. In the simple task with dots, subjects were told to press the right button to all four dots. As in Experiments 2 and 3, 4 catch trials occurred in each block of 24 reaction-time trials.

Second, each subject performed the choice task

Figure 4. Mean reaction times in simple (A) and choice (B) tasks with dots and letters as stimuli. (Memory load is the parameter.)

with one set of materials and the simple task with the other. Sixteen subjects performed the choice task with letters and the simple task with dots, and 16 performed the choice task with dots and the simple task with letters. Within each of these groups of 16 subjects, half began with the choice task and half began with the simple task. As before, subjects performed two blocks with one task (one with memory load and one without) before moving on to the next.

Third, the instructions for the two reaction-time tasks were given separately. When instructed in detail about the first task, subjects were simply told there would be another reaction-time task later. Detailed instructions about the second task were given only after they completed the first task.

\section{Results}

Reaction-time tasks. Each subject completed 24 trials with each task with and without a memory load. Mean reaction times in each of these combinations were computed for each subject and submitted to an analysis of variance. The means across subjects appear in Figure 4.

1. Between-subjects within-materials analyses. With letters as stimulus materials, subjects who performed the choice task took significantly longer than subjects who performed the simple task, $F(1,30)$ $=48.89, p<.01$, and overall, reaction time was longer with a memory load than without, $F(1,30)=65.07, p<.01$. As expected, the memory-load effect for subjects performing the choice task was significantly larger than the one for subjects performing the simple task, $F(1,30)=$ $5.17, p<.05$. 
Table 5

Proportion of Errors for Each Reaction-Time Task and Recall Accuracy in Each Condition of the Memory Task for Each Group of Subjects in Experiment 4

\begin{tabular}{lcccc}
\hline & & \multicolumn{3}{c}{ Reaction-time task } \\
\cline { 3 - 5 } Group & $\begin{array}{c}\text { Memory } \\
\text { load }\end{array}$ & None & \multicolumn{1}{c}{$\mathrm{A}$} & \multicolumn{1}{c}{$\mathrm{B}$} \\
\hline A-dots & 0 & - & .023 & .094 \\
B-letters & 8 & $-(5.67)$ & .031 & .154 \\
& & & $(5.47)$ & $(5.38)$ \\
A-letters & 0 & - & .044 & .036 \\
B-dots & 8 & $-(5.04)$ & .031 & .036 \\
& & & $(5.13)$ & $(5.03)$ \\
\hline
\end{tabular}

Note. Simple $=\mathrm{A}$; choice $=\mathrm{B}$. Recall accuracy is in parentheses.

With dots as stimulus materials, subjects performing the choice task did not take longer than subjects performing the simple task, $F(1,30)=1.57$. The overall effect of memory load was highly significant, $F(1,30)=79.62, p<.01$, and the interaction between memory load and tasks approached significance, $F(1,30)=3.38$, $p<.08$. This time, however, subjects performing the choice task seemed to suffer less from loading than subjects performing the simple task.

2. Within-subjects between-materials analyses. Subjects who performed the choice task with letters and the simple task with dots were slower with the choice task than with the simple task, $F(1,15)=91.89$, $p<.01$, and were slower with a memory load than without, $F(1,15)=55.07, p<$ .01 . The interaction between memory load and tasks was of the expected form-the choice task suffered more from loading than the simple task-but only approached significance, $F(1,15)=3.92, p<.07$.

Subjects who performed the choice task with dots and the simple task with letters were marginally slower with the choice task, $F(1,15)=3.98, p<.07$. They were considerably slower with a memory load than without, $F(1,15)=71.54, p<.01$, by about the same amount with each task; the interaction was not significant, $F(1,15)$ $=1.33$.

Error data for each task appear in Table 5.
Memory task. Each subject in each group recalled 24 eight-digit lists in singleand dual-task conditions. Means in each condition were computed for each subject, and means across subjects appear in Table 5. Note that single-task data are presented separately for each group.

1. Between-subjects within-materials analyses. With letters as stimuli in the reaction-time tasks, there were no differences in recall between the group receiving the choice task and the group receiving the simple task, $F(1,30)=1.47$, nor between single- and dual-task conditions, $F(1,30)$ $=1.07$. There was a suggestion that for the choice group, single-task recall was better than dual-task recall, whereas for the simple group, the opposite held. The interaction, however, only approached significance, $F(1,30)=3.51, p<.08$.

With dots as stimuli in the reaction-time tasks, there were no significant differences between groups or conditions.

2. Within-subjects between-materials analyses. Subjects who performed the simple task with dots and the choice task with letters recalled more in single-task conditions than in dual-task conditions, $F(1,30)=7.38, p<.05$, but no more with the simple task than with the choice task. Subjects who performed the simple task with letters and the choice task with dots recalled about the same amount in all conditions.

\section{Discussion}

In this experiment, consistent mapping in each task was ensured by having differen $t$ subjects perform different tasks with the same materials and by having the same subjects perform different tasks with different materials. This procedure was expected to produce interactions between memory load and task complexity, reflecting the adoption of differentially complex mapping rules for the different tasks. This expectation was confirmed for comparisons involving the choice task with letters but not for comparisons involving the choice task with dots. Subjects who performed the choice task with letters and 
the simple task with dots tended to suffer more from memory load in the choice task than in the simple task, and their choice-task performance suffered more from memory load than the simple-task performance of subjects who performed the simple task with letters. The latter result replicates Experiment 3.

Subjects who performed the choice task with dots and the simple task with letters suffered no more from memory load in the choice task than in the simple task, and their choice-task performance suffered no more than the simple-task performance of subjects who performed the simple task with dots. Most likely, this occurred because $S_{-} R$ relations in the choice task with dots had a higher degree of ideomotor compatibility than $S-R$ relations in the choice task with letters. Greenwald (1970, 1972; Greenwald \& Shulman, 1973) has argued that $\mathrm{S}-\mathrm{R}$ relations are ideomotor compatible if the sensory effects of the stimuli resemble the sensory consequences of their corresponding responses, and that such relations allow economical translation from the stimulus code to the response code (i.e., only one production is required for the whole task: $\mathrm{El}: \quad(\mathrm{EYE}=\mathrm{X} \rightarrow$ HAND $=\mathrm{X}$ ), where $\mathrm{X}$ is the stimulus code and the response code). In particular, he has argued that tasks like the present choice task with dots in which stimuli on one side of the body's midline are mapped onto responses on the same side are ideomotor compatible, and has demonstrated that increases in ideomotor compatibility reduce choice reaction time (Greenwald, 1970) and reduce dual-task interference (Greenwald, 1972; Greenwald \& Shulman, 1973). The present results in the choice task with dots corroborate these findings.

\section{General Discussion}

The major results can be summarized as follows: A concurrent memory load produced more interference with a complex task than with a simple one when the different tasks were performed by different subjects, and tended to do so when the same subjects performed the different tasks with different materials. But when the same subjects performed the different tasks with the same materials, memory-load interference did not vary with task complexity. At an empirical level, this suggests that memory-load interference is not restricted to any particular stage of processing (i.e., the stages differentiating choice and simple tasks), since interactions appeared and disappeared in different experiments, and that short-term memory is not the only resource demanded by reaction-time tasks, since complex tasks took longer than simple ones even when they produced no more memory-load interference. These conclusions were also drawn from the analysis of previous findings, summarized in Table 1, and extend their generality. Clearly, interactions between memory load and task complexity are not obligatory, but rather, they depend on the subject's strategy.

The experiments also addressed two interpretations of the view that memoryload interference reflects the memory demands of retaining a novel organization of abilities required for the task, one in terms of traditional stage theory and one in terms of S-R mapping rules. The stagetheory interpretation asserts that processing stages are held in memory to enable performance and predicts more interference whenever one task involves more stages than another. This interpretation is consistent with the interactions observed in Experiment 3 and 4 but is ruled out by the additivity found in Experiments 1 and 2 . However, the traditional stages may still underly performance if they are controlled by remembering something other than stages, or stages defined by some more flexible theory may both underly and control performance.

In contrast with traditional stage theory, the S-R mapping interpretation asserts that sets of $S-R$ mapping rules are held in memory to enable performance and predicts more interference whenever one task involves more complex mapping rules than another. Previous research suggests that minimal mapping rules of different complexity for different tasks will be developed 
under consistent mapping conditions, like those in Experiments 3 and 4, and that one complex set of mapping rules to handle all tasks will be developed under varied mapping conditions, like those in Experiments 1 and 2. The interactions in Experiments 3 and 4 and the additivity in Experiments 1 and 2 support this interpretation.

Note that the findings support the conclusion that mapping rules are held in memory but do not necessarily indicate that the mapping rules actually enable performance. This assumption cannot be tested by comparing memory-load interference, since it requires demonstrating that performance cannot occur without the mapping rules in memory, but it is central to the interpretation offered here. Clearly, verbal instructions enabled performance on the tasks studied here in some way; my speculation is that the propositional content of the instructions is reduced to a set of productions representing the required mapping rules, which are held in memory and applied when their conditions are satisfied. Presumably, with extended practice, the productions are transferred to long-term memory.

A general implication of this view is that memory-load interference can be used to investigate strategies in a variety of contexts. The $S-R$ mapping rules held in memory represent a compromise between the structure described in the instructions (i.e., the conditions and actions they specify), the structure of the subject's information-processing system (i.e., the abilities brought to the task), and the structure of the task environment (i.e., which conditions and actions it affords). Presumably, a number of these compromises or strategies will be attempted until an optimal one is found (Logan, 1979). The present research suggests that changes in strategy may be observable as changes in memory-load interference. Moreover, if the possible strategies are understood well enough to be described in a way that their respective demands on memory can be estimated (i.e., as production systems or some suitable formalism), memory-load interference may provide a means to distinguish among them. Given the importance now placed on the strategic aspects of performance (e.g., Newell \& Simon, 1972; Posner, 1978), these applications may be valuable.

Finally, the conclusion that short-term memory retains sets of mapping rules to enable performance on reaction-time tasks is consistent with my earlier interpretation of memory-load interference in reactiontime tasks as reflecting the attention demands of preparation (Logan, 1978, 1979). From the point of view of capacity theory, attention and short-term memory may be the same thing; both are central in the architecture of the informationprocessing system, both have limited capacities that can be allocated strategically, and both have been implicated in the control of behavior (cf. Kahneman, 1973; Newell, 1973). Moreover, preparation and the maintenance of what has been prepared is the general case of arriving at a set of $\mathrm{S}-\mathrm{R}$ mapping rules and retaining them in memory. Thus, the present findings may also support the earlier conclusion that the primary function of attention in reactiontime tasks is to prepare and maintain a set to perform the task. This conclusion has roots in the early introspective studies of reaction time (see Woodworth, 1938) and is supported today in a variety of contexts in which attention is studied. Gottsdanker's (1979) demonstration that a psychological refractory period can be produced by a stimulus that is expected but not presented is an elegant example.

\section{Reference Note}

1. Logan, G. D. Attention demands of encoding and comparison in visual search. Paper presented at the annual meeting of the Canadian Psychological Association, Toronto, Ontario, June 1976.

\section{References}

Crowder, R. G. Short-term memory for words with a perceptual-motor interpolated activity. Journal of Verbal Learning and Verbal Behavior, 1967, 6, 753-761.

Doll, T. J. Short-term retention: Preparatory set as covert rehearsal. Journal of Experimental Psychology, 1969, 82, 175-182. 
Donders, F. C. On the speed of mental processes. In W. G. Koster (Ed. and trans.), Attention and performance II. Amsterdam: North Holland, 1969. (Orginally published, 1868.)

Egeth, H. Attention and preattention. In G. $H$. Bower (Ed.), The psychology of learning and motivation (Vol. 11). New York: Academic Press, 1977.

Gottsdanker, R. A psychological refractory period or an unprepared period? Journal of Experimental Psychology: Human Perception and Performance, $1979,5,208-215$.

Greenwald, A. G. A choice reaction time test of ideomotor theory. Journal of Experimental Psychology, 1970, 86, 20-25.

Greenwald, A. G. On doing two things at once: Time sharing as a function of ideomotor compatibility. Journal of Experimental Psychology, 1972, 94, 52-57.

Greenwald, A. G., \& Shulman, H. G. On doing two things at once: II. Elimination of the psychological refractory period effect. Journal of Experimental Psychology, 1973, 101, 70-76.

Kahneman, D. Attention and effort. Englewood Cliffs, N. J. : Prentice-Hall, 1973.

Keele, S. W., \& Boies, S. J. Processing demands of sequential information. Memory $\&$ Cognition, $1973,1,85-90$.

Klapp, S. T. Short-term memory as a response preparation state. Memory \& Cognition, 1976, 4, 721-729.

Logan, G. D. Attention in character-classification tasks : Evidence for the automaticity of component stages. Journal of Experimental Psychology: General, 1978, 107, 32-63.

Logan, G. D. On the use of a concurrent memory load to measure attention and automaticity. Journal of Experimental Psychology: Human Perception and Performance, 1979, 5, 189-207.

Newell, A. Production systems: Models of control structures. In W. G. Chase (Ed.), Visual information processing. New York: Academic Press, 1973.

Newel, A., \& Simon, H. A. Human problem solving. Englewood Cliffs, N.J.: Prentice-Hall, 1972.

Posner, M. I. Chronometric explorations of mind. Hillsdale, N.J. : Erlbaum, 1978.

Posner, M. I., \& Boies, S. J. Components of attention. Psychological Review, 1971, 78, 391-408.

Shulman, H. G., \& Greenberg, S. N. Perceptual deficit due to division of attention between memory and perception. Journal of Experimental Psychology, 1971, 88, 171-176.

Shulman, H. G., Greenberg, S. N., \& Martin, J. Intertask delay as a parameter of perceptual deficit in divided attention. Journal of Experimental Psychology, 1971, 88, 439-440.

Smith, E. E. Choice reaction time: An analysis of the major theoretical positions, Psychological Bulletin, 1968, 69, 77-110.

Sternberg, S. Memory scanning: Mental processes revealed by reaction-time experiments. American Scientists, 1969, 57, 421-457.

Woodworth, R. S. Experimental psychology. New York: Holt, 1938.

Received February 12, 1979 